EPJ Web of Conferences 21, 06002 (2012)

DOI: $10.1051 /$ epjconf/20122106002

(c) Owned by the authors, published by EDP Sciences, 2012

\title{
Population of rotational bands in superheavy nuclei
}

\author{
A.S. Zubov ${ }^{1}$, V.V. Sargsyan ${ }^{1,2}$, G.G. Adamian ${ }^{1,3}$, and N.V. Antonenko ${ }^{1}$ \\ 1 Joint Institute for Nuclear Research, 141980 Dubna, Russia \\ 2 Yerevan State University, 0025 Yerevan, Armenia \\ 3 Institute of Nuclear Physics, Tashkent 702132, Uzbekistan
}

\begin{abstract}
Using the statistical approach, we study the population of ground-state rotational bands of superheavy nuclei produced in the fusion-evaporation reactions ${ }^{208} \mathrm{~Pb}\left({ }^{48} \mathrm{Ca}\right.$, $2 n)^{254} \mathrm{No},{ }^{206} \mathrm{~Pb}\left({ }^{48} \mathrm{Ca}, 2 n\right){ }^{252} \mathrm{No}$, and ${ }^{204} \mathrm{Hg}\left({ }^{48} \mathrm{Ca}, 2 n\right){ }^{250} \mathrm{Fm}$. We calculate relative intensities of $E 2$-transitions between the rotational states and entry spin distributions of the residual nuclei, evaporation residue cross sections, and excitation functions for these reactions. Fermi-gas model is used for the calculation of level density, and damping of shell effects both with excitation energy and angular momentum is taking into account. The results are in a good agreement with the experiment data.
\end{abstract}

\section{Introduction}

The existence of heaviest nuclei with $Z \geq 100$ is mainly determined by their shell structure, as the liquid drop part of their fission barriers vanishes with the growth of charge number. There is a variety of theoretical approaches which predict the next doubly magic shell closure beyond ${ }^{208} \mathrm{~Pb}$. Reaching the region of superheavy nuclei in the vicinity of this closure, so called "island of stability", is a challenge for the modern nuclear physics. The macroscopic-microscopic approaches predict the magic nucleus with $Z=114$ [1]. The closed shell $Z=114$ disappears in the framework of self-consistent mean-field models [2-6] which result the strong shell effects at $Z=120-126$. Although all these models provide close binding energies for nuclei with $Z \leq 114$, their predictions for the spins of the ground states, quasiparticle spectra, and for the moments of inertia are rather different. Therefore, one can test the theoretical models by comparing their structure predictions with the spectroscopical data.

Experimental study of rotational bands of superheavies is especially hopeful, as the limits of stability of these nuclei in spin and excitation energy are governed by the fission barrier, which is mostly defined by shell component. The investigation of high-spin spectra also provides information about angular momentum dependence of the shell effects. Unfortunately, there are still a little experimental data for the heaviest evaporation residues, as their production cross sections are too small for the spectroscopical analysis. However, using the in-beam studies, the structure of high-spin excited states up to $\sim 20 \hbar$ has been recently identified for the nuclei ${ }^{254} \mathrm{No},{ }^{252} \mathrm{No}$, and ${ }^{250} \mathrm{Fm}$, produced with the cross sections $0.2-3 \mu \mathrm{b}[7-10]$.

The nuclei studied are produced in fusion-evaporation reactions, which can be successfully described using the statistical approach. In Refs. [11-14] we have already calculated evaporation residue cross sections and excitation functions for superheavy isotopes using their properties, predicted by different theoretical models [15-18]. The results are in a good agreement with the experimental data.

In the present paper, we extend the statistical approach to describe the population of yrast rotational states in the residual superheavy nuclei and the production of evaporation residues in the complete fusion reactions ${ }^{206,208} \mathrm{~Pb}\left({ }^{48} \mathrm{Ca}, 2 n\right){ }^{252,254} \mathrm{No}$ and ${ }^{204} \mathrm{Hg}\left({ }^{48} \mathrm{Ca}, 2 n\right){ }^{250} \mathrm{Fm}$, taking into consideration the survival of compound nuclei against fission at different angular momenta. The relative intensities of E2-transitions and entry spin distributions of the residual nuclei can be derived from the calculated population cross sections of the rotational states and compared with the experimental data [7-10].

This is an Open Access article distributed under the terms of the Creative Commons Attribution-Noncommercial License 3.0, which permits unrestricted use, distribution, and reproduction in any noncommercial medium, provided the original work is properly cited. 
Using the same approach and set of parameters, we also calculate evaporation residue cross sections for these reactions.

\section{Model}

\subsection{Formation of yrast residual nucleus}

Our model treats the formation of yrast residual nucleus as a three-step process. First, the rotating dinuclear system is formed in the entrance channel by the transition of the colliding nuclei over the entrance (Coulomb) barrier. Second, this dinuclear system evolves into the compound nucleus in the mass (charge) asymmetry coordinate. Third, the formed excited rotating compound nucleus transforms to the yrast residual nucleus by the emission of the neutrons and statistical $\gamma$-quanta. The population of yrast rotational state $L^{+}$with spin $L$ and positive parity depends on survival of the compound nucleus against fission in $x n$ evaporation channel. The residual excitation energy $\Delta E^{*}$ after the emission of neutrons should be less than the fission barrier and quite small to be taken away by $k$ statistical dipole $\gamma$-quanta ( $k$ is a number of statistical $E 1$-transitions).

Thus, the population cross section of the state $L^{+}$depends on the partial capture cross section $\sigma_{c}$, on the probability $P_{C N}$ of the compound nucleus formation after the capture and on the survival probability $W_{\text {sur }}^{\text {pop }}$ of the excited compound nucleus against fission in the $x n k \gamma$ evaporation channel as the following:

$$
\sigma_{p o p}\left(E_{\mathrm{c} . \mathrm{m} .}, L\right)=\sum_{k} \sigma_{c}\left(E_{\mathrm{c} . \mathrm{m} .}, J\right) P_{C N}\left(E_{\mathrm{c} . \mathrm{m} .}, J\right) W_{s u r}^{p o p}\left(E_{C N}^{*}, J, \Delta E^{*}\right)
$$

where $J=L+k l_{g}+x l_{n}$ is the initial angular momentum of compound nucleus. Average angular momentum carried out by neutrons and $E 1$ statistical $\gamma$-quanta was taken $l_{n}=0.5$ and $l_{g}=1$, respectively. Since the bombarding energy $E_{\text {c.m. }}$ is usually higher than the $Q$ value for the compound nucleus formation, the produced nucleus has thermal excitation energy

$$
E_{C N}^{*}(J)=E_{\text {c.m. }}-Q-\hbar^{2} J(J+1) /(2 \mathfrak{J}),
$$

where $\mathfrak{I}$ is the moment of inertia of the compound nucleus. In our calculations this value is extracted from the experimental data [7-10] and taken constant at each evaporation step. In the first evaporation step the mean values of excitation energy $E_{1}^{*}=E_{C N}^{*}(J)$ and angular momentum $J_{1}=J$, in the second one $E_{2}^{*}=E_{1}^{*}-2 T$ and $J_{2}=J-0.5$, where $T=\sqrt{E_{C N}^{*}(J) / a_{C N}}$ is the nuclear temperature. The survival probability in the case of the evaporation of $x$ neutrons and $k \gamma$-quanta is calculated as

$$
W_{\text {sur }}^{\text {pop }}\left(E_{C N}^{*}, J, \Delta E^{*}\right)=P_{x n k \gamma}\left(E_{C N}^{*}, J, \Delta E^{*}\right) \prod_{i=1}^{x} \frac{\Gamma_{n}\left(E_{i}^{*}, J_{i}\right)}{\Gamma_{f}\left(E_{i}^{*}, J_{i}\right)},
$$

where $P_{x n k \gamma}$ is the probability of realization of an $x n k \gamma$ channel at a given $E_{C N}^{*}$ and $J$ and $\Gamma_{n}\left(E_{i}^{*}, J_{i}\right)$ and $\Gamma_{f}\left(E_{i}^{*}, J_{i}\right)$ are, respectively, the widths of neutron emission and fission in the evaporation step $i$ and $P_{x n k \gamma}$ is the probability of realization of an $x n k \gamma$ channel at a given $E_{C N}^{*}$ and $J$. In other words, $P_{x n k y}$ gives the probability to emit $x$ neutrons with quite large kinetic energy to cool the nucleus to $E^{*}<\Delta E^{*}$, and, afterwards, to reach the $L^{+}$state by the sequence of $k E 1$-transitions.

The relative intensity of $E 2$ multipolarity transitions between the yrast rotational states $L^{+}$and $(L-2)^{+}$can be calculated as

$$
I(L \rightarrow L-2)=\frac{\sum_{x=L / 2}^{\left[L_{\max } / 2\right]} \sigma_{p o p}\left(E_{\mathrm{c} . \mathrm{m} .}, 2 x\right)}{\sum_{x=0}^{\left[L_{\max } / 2\right]} \sigma_{p o p}\left(E_{\mathrm{c} . \mathrm{m} .}, 2 x\right)},
$$

where $L_{\max }$ is the maximum spin of states, which can be populated at the certain beam energy $E_{\mathrm{c} . \mathrm{m}}$. 


\section{CNR*11}

\subsection{Emission of statistical gamma-quanta}

The probability to emit statistical $E 1 \gamma$-quantum with the energy in the interval from $\epsilon_{\gamma}$ to $\epsilon_{\gamma}+d \epsilon_{\gamma}$ at the fixed excitation energy $E^{*}$ is calculated as

$$
v\left(E^{*}, \epsilon_{\gamma}\right) d \epsilon_{\gamma}=\frac{\epsilon_{\gamma}^{3} f_{E 1}\left(\epsilon_{\gamma}\right) \rho\left(E^{*}-\epsilon_{\gamma}\right)}{\int_{0}^{E^{*}} \epsilon_{\gamma}^{\prime 3} f_{E 1}\left(\epsilon_{\gamma}^{\prime}\right) \rho\left(E^{*}-\epsilon_{\gamma}^{\prime}\right) d \epsilon_{\gamma}^{\prime}} d \epsilon_{\gamma},
$$

where $\gamma$ strength function $f_{E 1}\left(\epsilon_{\gamma}\right)$ is taken in Lorenzian form, as in Ref. [19]

$$
f_{E 1}\left(\epsilon_{\gamma}\right)=3.31 \times 10^{-6} \frac{Z(A-Z)}{A} \frac{\epsilon_{\gamma} \Gamma_{G D R}}{\left(E_{G D R}^{2}-\epsilon_{\gamma}^{2}\right)^{2}+\epsilon_{\gamma}^{2} \Gamma_{G D R}^{2}} .
$$

Here, $E_{G D R}=167.23 A^{-1 / 3}\left(1.959+14.074 A^{-1 / 3}\right)^{-1 / 2} \mathrm{MeV}$ is the GDR energy, and $\Gamma_{G D R}=5 \mathrm{MeV}$ is the GDR width [19].

After the evaporation of 2 neutrons the excitation energy of the residual nucleus is small in the reactions considered, and to calculate the level density, one should use the model with the constant temperature [20] (see Sect. 2.6). If $\epsilon_{\gamma} \ll E_{G D R}, f_{E 1}\left(\epsilon_{\gamma}\right) \approx$ const $\times \epsilon_{\gamma}$, and, in the case of $E^{*} \gg T_{0}$, one can obtain an analytical expression for the vague $v\left(\epsilon_{\gamma}\right)$

$$
v\left(\epsilon_{\gamma}\right) d \epsilon_{\gamma} \approx \frac{\epsilon_{\gamma}^{4} \exp \left[-\epsilon_{\gamma} / T_{0}\right]}{24 T_{0}^{5}} d \epsilon_{\gamma} .
$$

The condition $\frac{\partial v\left(\epsilon_{\gamma}\right)}{\partial \epsilon_{\gamma}}=0$ gives us the most probable energy $\epsilon_{\gamma}=4 T_{0}$ of $E 1 \gamma$-quantum. If $E^{*} \leq 4 T_{0}$, $\gamma$-quantum carries out almost all the excitation energy. Therefore, we take $\Delta E^{*}=4 T_{0} \approx 1.6 \mathrm{MeV}$ at the calculation of the probability $w_{2 n k \gamma}$ for all nuclei considered.

The probability $P_{x n k \gamma}$ can be calculated using the sequent folding procedure of the Maxwellian neutron spectra and the statistical $E 1 \gamma$-quantum energy distributions, given by Eq. 5 [21].

\subsection{Evaporation residue cross section}

In fusion-evaporation reactions the partial evaporation residue cross section at angular momentum $J[11,22,23]$

$$
\sigma_{E R}\left(E_{\text {c.m. }}, J\right)=\sigma_{c}\left(E_{\text {c.m. }}, J\right) P_{C N}\left(E_{\text {c.m. }}, J\right) W_{\text {sur }}\left(E_{\text {c.m. } .} J\right),
$$

where

$$
W_{\text {sur }}\left(E_{C N}^{*}, J\right)=P_{x n}\left(E_{C N}^{*}\right) \prod_{i=1}^{x} \frac{\Gamma_{n}\left(E_{i}^{*}, J_{i}\right)}{\Gamma_{f}\left(E_{i}^{*}, J_{i}\right)},
$$

is the survival probability of the excited compound nucleus against fission in $x n$ evaporation channel $[24,25]$. The probability of realization of an $x \mathrm{n}$ channel at a given $E_{C N}^{*}$, Gaussian-like function, is calculated, as in Ref. [26].

Using Eq. (2), one can define the maximal value of angular momentum in the fusion-evaporation reaction at $x n$ evaporation channel from the condition

$$
E_{\text {c.m. }}-Q-\sum_{i=1}^{x} B_{n}(i)-\hbar^{2} J_{\max }\left(J_{\max }+1\right) /(2 \mathfrak{J})>0 .
$$

Then, the total evaporation residue cross section is

$$
\sigma_{E R}\left(E_{\mathrm{c} . \mathrm{m} .}\right)=\sum_{J=0}^{J_{\max }} \sigma_{E R}\left(E_{\mathrm{c} . \mathrm{m} .}, J\right) .
$$


In Refs. [11-14] another approximate expression was used for the calculation of the evaporation residue cross section:

$$
\sigma_{E R}\left(E_{\mathrm{c} . \mathrm{m} .}\right) \approx \sigma_{c}\left(E_{\mathrm{c} . \mathrm{m} .}\right) P_{C N}\left(E_{\mathrm{c} . \mathrm{m} .}, J=0\right) W_{\text {sur }}\left(E_{\mathrm{c} . \mathrm{m} .}, J=0\right),
$$

where the dependence on angular momenta is taken into account only in the effective capture cross section $\sigma_{c}\left(E_{\mathrm{c} . \mathrm{m} .}\right)=\pi \hbar^{2}\left(J_{\max }+1\right)^{2} T\left(E_{\mathrm{c} . \mathrm{m} .}\right), T\left(E_{\mathrm{c} . \mathrm{m} .}\right)$ is a transition coefficient through the entrance Coulomb barrier, calculated in the parabolical approximation, and $J_{\max }=10-15$. Eq. (12) was derived assuming a sharp decrease of $W_{\text {sur }}\left(E_{\mathrm{c} . \mathrm{m} .}, J\right)$ with increasing $J$.

\subsection{Capture cross section}

The partial capture cross section $\sigma_{c}\left(E_{\mathrm{c} . \mathrm{m} .}, J\right)$ depends on the capture probability $P_{\text {cap }}$ which is related to the transition through the entrance barrier:

$$
\sigma_{c}\left(E_{\mathrm{c} . \mathrm{m} .}, J\right)=\frac{\pi \hbar^{2}}{2 \mu E_{\mathrm{c} . \mathrm{m} .}}(2 J+1) P_{\mathrm{cap}}\left(E_{\mathrm{c} . \mathrm{m} .}, J\right) .
$$

Here, $\mu$ is the reduced mass of relative motion. The capture probability $P_{\text {cap }}$ is calculated using the quantum diffusion approach based on the formalism of reduced density matrix [27]. Many quantummechanical, dissipative effects and non-Markovian effects accompanying the passage through the potential barrier are taken into consideration in this formalism.

The dependence of the capture cross section on the angular momentum is shawn in Fig. 1 for

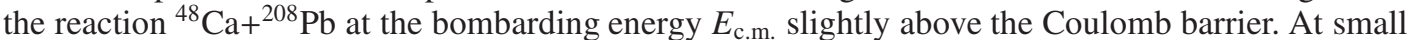
angular momenta, the function $\sigma_{c}\left(E_{\mathrm{c} . \mathrm{m}}, J\right)$ growthes due to the factor $2 J+1$ in Eq. (13), while the decrease of the capture probability $P_{\text {cap }}$ caused by the increase of the centrifugal term in the nucleusnucleus potential, which leads to the higher potential barrier in the entrance channel, is rather weak. At larger $J$, the system of colliding nuclei turns out in the sub-barrier region and the capture cross section falls down, since in this region the decrease of $P_{\text {cap }}$ with $J$ is not compensated by the factor $2 J+1$. The larger value $E_{\mathrm{c} . \mathrm{m}}$, the larger value of angular momentum, at which the transition to the sub-barrier region occurs.

\subsection{Complete fusion probability}

The probability $P_{C N}$ of the compound nucleus formation depends on the competition between complete fusion and quasifission at the complete fusion stage of the reaction. This value is calculated using dinuclear system model, as in Refs. [11,23]. For all the reactions considered at given beam energies and interval of angular momenta the dependence of $P_{C N}$ on $E_{\mathrm{c} . \mathrm{m}}$., and $J$ can be neglected and $P_{C N}\left(E_{\mathrm{c} . \mathrm{m} .}, J\right) \approx P_{C N}\left(E_{\mathrm{c} . \mathrm{m} .}, J=0\right) \approx 0.5$.

\subsection{Calculation of channel widths and level density}

The decay width of channel $i$ is given in terms of the probability $R_{C N_{i}}$ of this process as [24,25,28-31, 20]

$$
\Gamma_{i}=\frac{R_{C N_{i}}}{2 \pi \rho\left(E_{C N}^{*}, J\right)} .
$$

The probability of neutron emission is calculated as

$$
R_{C N_{n}}\left(E_{C N}^{*}, J\right)=\sum_{j=J-1 / 2}^{j=J+1 / 2}\left(\int_{0}^{E_{C N}^{*}-B_{n}} \rho_{d}\left(E_{C N}^{*}-B_{n}-\epsilon, j\right) T_{J}(A-1, \epsilon) d \epsilon\right),
$$




\section{$\mathrm{CNR} * 11$}

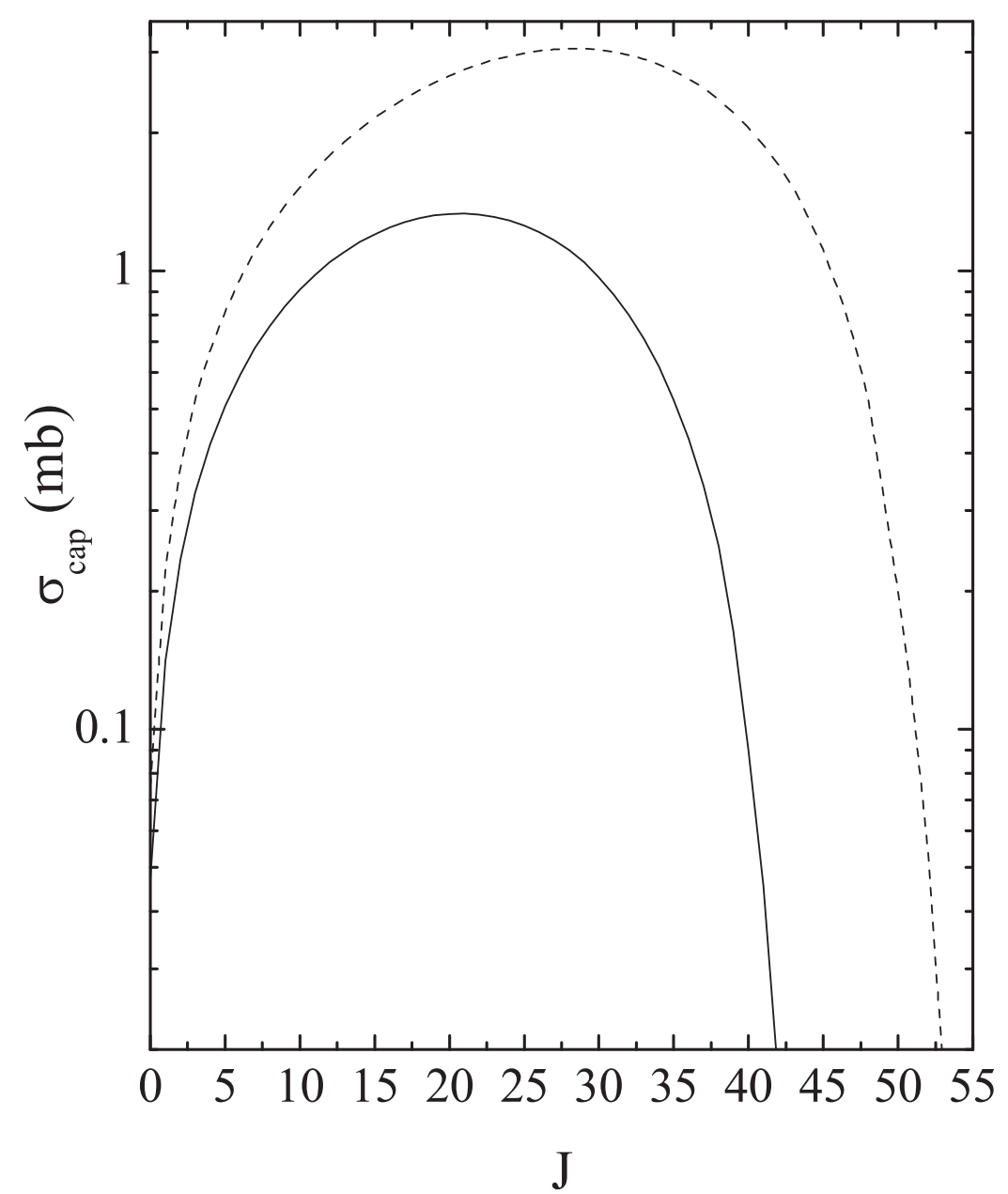

Fig. 1. Capture cross section as a function of angular momentum for the ${ }^{48} \mathrm{Ca}+{ }^{208} \mathrm{~Pb}$ reaction at beam energy 215 $\mathrm{MeV}$ (solid curve) and $219 \mathrm{MeV}$ (dashed curve).

where $\rho_{d}\left(E_{C N}^{*}-B_{n}-\epsilon, j\right)$ is the level density of the daughter nucleus. The experimental values [32] of neutron binding energies $B_{n}$ are used, and the transition coefficients $T_{J}(A-1, \epsilon)$ are calculated as in Ref. [33].

The fission probability in the case of an one-hump barrier of height $B_{f}\left(E_{C N}^{*}, J\right)$ and curvature determined by $\hbar \omega$ is given as

$$
R_{C N_{f}}\left(E_{C N}^{*}, J\right)=\int_{0}^{E_{C N}^{*}-B_{f}\left(E_{C N}^{*}, J\right)} \frac{\rho_{f}\left(E_{C N}^{*}-B_{f}\left(E_{C N}^{*}, J\right)-\epsilon, J\right) d \epsilon}{1+\exp \left[2 \pi\left(\epsilon+B_{f}\left(E_{C N}^{*}, J\right)-E_{C N}^{*}\right) /(\hbar \omega)\right]},
$$

where $\rho_{f}\left(E_{C N}^{*}-B_{f}\left(E_{C N}^{*}, J\right)-\epsilon, J\right)$ is the level density at the saddle point. For all the nuclei considered, we take $\hbar \omega=2.2 \mathrm{MeV}$.

In the simplest way the level density is calculated with the Fermi-gas model [31] as

$$
\rho\left(E_{C N}^{*}, J\right)=\frac{2 J+1}{24 \sqrt{2} \sigma^{3} a^{1 / 4}\left(E_{C N}^{*}-\delta\right)^{5 / 4}} \exp \left\{2 \sqrt{a\left(E_{C N}^{*}-\delta\right)}\right\},
$$

with excitation energy $E_{C N}^{*}(J)$ defined in (2) and $\sigma^{2}=6 \overline{m^{2}} \sqrt{a\left(E_{C N}^{*}-\delta\right)} / \pi^{2}$. The pairing correction $\delta$ is set $\delta=0.7 \approx 12 / \sqrt{A}, 0$ and $-0.7 \mathrm{MeV}$ for even-even, odd, and odd-odd nuclei, respectively. The 
EPJ Web of Conferences

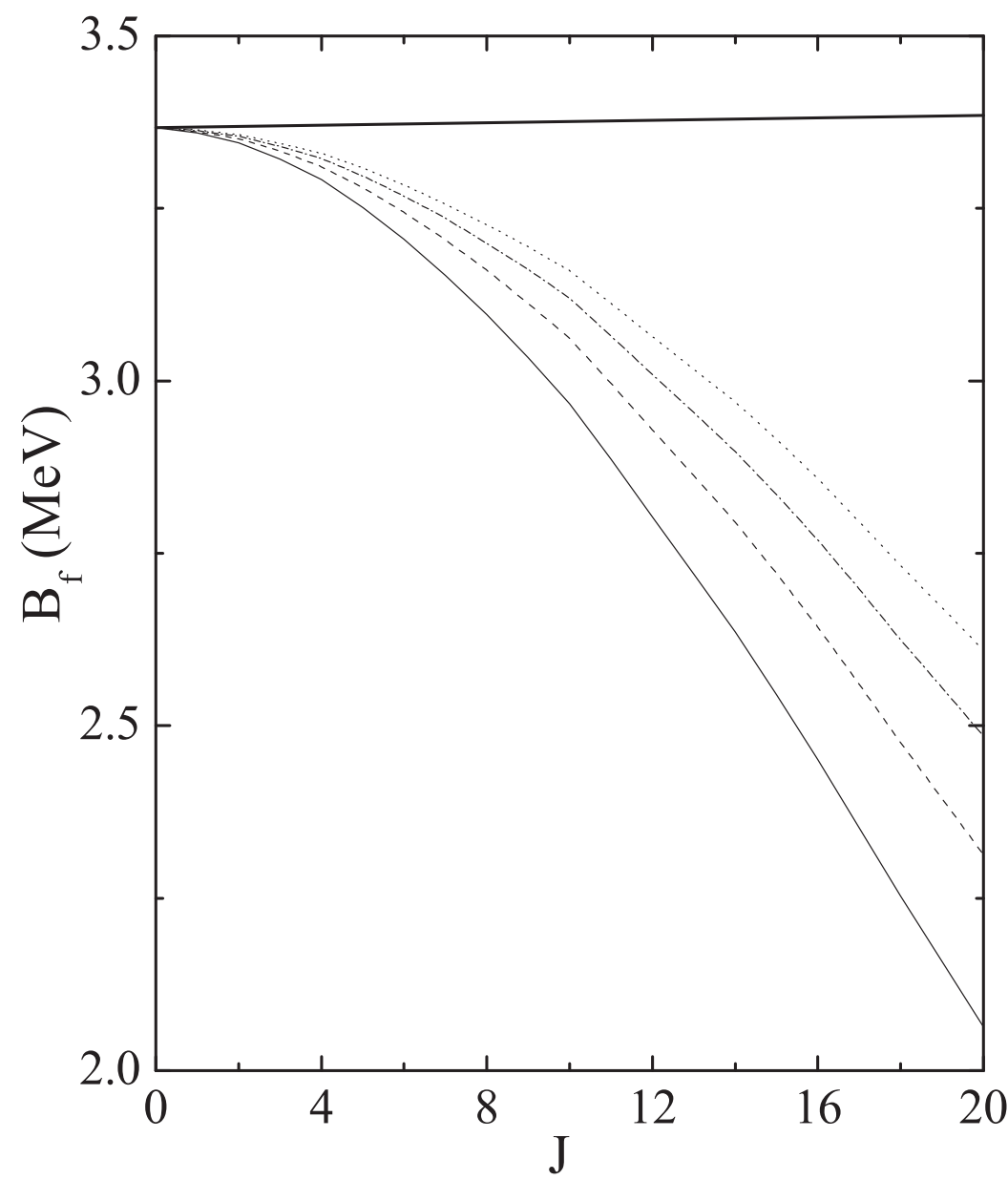

Fig. 2. The calculated dependence of the fission barrier $B_{f}$ on angular momentum for ${ }^{256}$ No. The excitation energy $E_{C N}^{*}=19.3 \mathrm{MeV}$ at $J=0$. Solid, dashed, dashed-dot, and dotted curves correspond to $D=600,800,1000$, and 1200, respectively. The dependence $B_{f}$ on $J$ without taking into consideration damping of shell effects with angular momentum is denoted by thick solid curve.)

level density parameter $a$ is proportional to the density of single-particle states at the Fermi surface. We take $a=A / 10 \mathrm{MeV}^{-1}$ for all considered nuclei. The average projection of the angular momentum of these states is estimated as $\overline{m^{2}} \approx 0.24 A^{2 / 3}$. For small excitation energies $E_{C N}^{*}-\delta<U_{x}=2.2 \mathrm{MeV}$ we calculate the level density using the model with constant temperature $T_{0}[20]$ as

$$
\rho\left(E_{C N}^{*}, J\right)=\frac{1}{2 \sqrt{2 \pi} \sigma^{3} T_{0}} \exp \left(\frac{E_{C N}^{*}-U_{0}}{T_{0}}\right) .
$$

The parameters $T_{0}$ and $U_{0}$ in (18) are defined to supply the smooth transition from (17) at $E_{C N}^{*}=U_{x}$ [20].

The fission barrier $B_{f}$ has the liquid drop and microscopical parts, $B_{f}^{L D}$ and $B_{f}^{M}$, respectively. The liquid drop part $B_{f}^{L D}(J)$ is calculated as in Ref. [34] for all considered nuclei. The value $B_{f}^{M}=$ $\delta W_{s d}^{A}-\delta W_{g r}^{A}$ is related to the shell correction $\delta W_{g r}^{A}$ of the nucleus with mass number $A$ at the ground state and the shell correction $\delta W_{s d}^{A}$ at the saddle point. Usually, one neglects the shell correction at the saddle point, $\delta W_{s d}^{A} \approx 0$ [35]. Thus, $B_{f}^{M}=B_{f}^{M}\left(E_{C N}^{*}=0\right) \approx\left|\delta W_{g r}^{A}\left(E_{C N}^{*}=0\right)\right|$. We use the predicted values of shell corrections from Ref. [18] for all considered nuclei. The dampings of the shell effects 
CNR*11

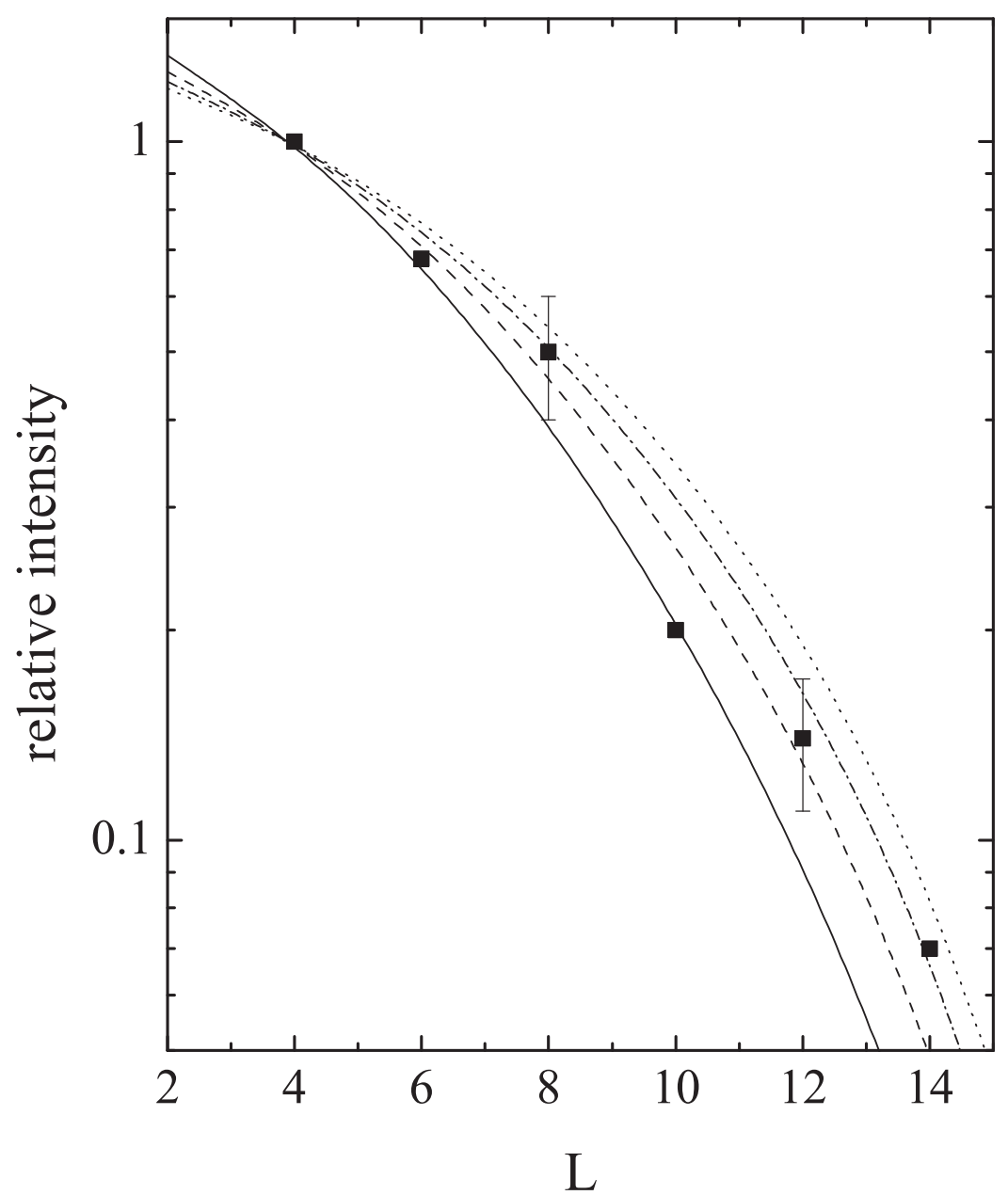

Fig. 3. Relative transition intensities in the yrast rotational band of ${ }^{254} \mathrm{No}$, produced in the reaction ${ }^{208} \mathrm{~Pb}\left({ }^{48} \mathrm{Ca}\right.$, $2 n)^{254} \mathrm{No}$ at the beam energy $215 \mathrm{MeV}$. The experimental data from Refs. [7,8] are presented by squares. The calculated points are connected by lines. Solid, dashed, dashed-dot and curves correspond to $D=600,800,1000$, and 1200 , respectively.)

with the growth of the excitation energy and angular momentum are taken into consideration. These dependencies are approximated by the exponential damping factors as

$$
B_{f}\left(E_{C N}^{*}, J\right)=B_{f}^{L D}(J)+B_{f}^{M}\left(E_{C N}^{*}=0\right) \exp \left[-E_{C N}^{*}(J) / E_{D}\right] \exp \left[-\hbar^{2} J(J+1) / D\right],
$$

where the damping parameter $D$ can be set to describe the experimental data [7-10]. In the calculations, we use the following expression for the damping parameter $E_{D}$ suggested in Ref. [19]

$$
E_{D}=\alpha_{0} A^{4 / 3} / a
$$

where $\alpha_{0}=0.4$.

In Fig. 2 the dependence of total fission barrier $B_{f}$ is presented for the compound nucleus ${ }^{256} \mathrm{No}$, produced in the reaction ${ }^{48} \mathrm{Ca}+{ }^{208} \mathrm{~Pb}$ with the beam energy $215 \mathrm{MeV}$. In this case, the excitation energy $E_{C N}^{*}=19.3 \mathrm{MeV}$ at $J=0$. The value $E_{C N}^{*}$ changes with $J$, according to Eq. (2), and damping of the shell effects with the excitation energy should be taken into account. However, this effect is small, as in the considered interval of angular momenta the variation of the excitation energy is much less than $E_{C N}^{*}(J=0)$, and it is compensated by the decrease of the liquid drop part of fission barrier with $J$. 
EPJ Web of Conferences

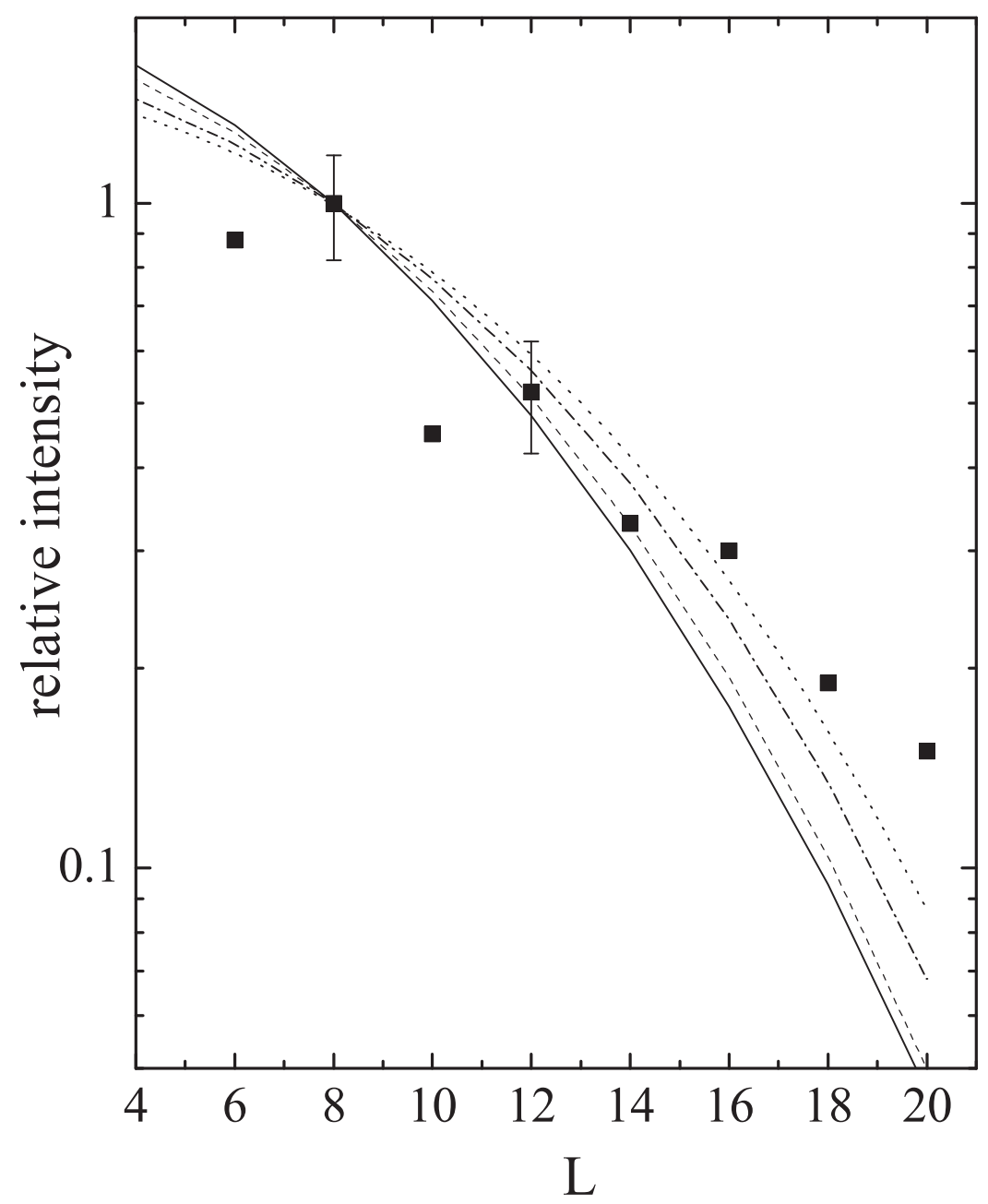

Fig. 4. The same as in Fig. 3, but for the beam energy $219 \mathrm{MeV}$.)

This is clearly seen from the dependence $B_{f}$ on $J$, calculated without taking into account damping of the shell effects with the angular momentum, which is also shown in Fig. 2 for the comparison. Thus, one can conclude that for the reactions considered the dependence of the fission barrier on angular momentum is mainly governed by the damping of the shell effects on angular momentum, $\exp [-J(J+1) / D]$.

\section{Calculated results}

\subsection{The residual nucleus ${ }^{254}$ No}

In Figs. 3-4 the measured and calculated relative transition intensities in the rotational band of the nucleus ${ }^{254} \mathrm{No}$, produced in the reaction ${ }^{208} \mathrm{~Pb}\left({ }^{48} \mathrm{Ca}, 2 n\right){ }^{254} \mathrm{No}$ are presented. The experiment was performed at the beam energies $215 \mathrm{MeV}$ [7] and $219 \mathrm{MeV}$ [8] that correspond to the excitation energies $E_{C N}^{*}=19.3$ and $22.7 \mathrm{MeV}$ at $J=0$, respectively, which are close to the maximum of the excitation function. The relative intensities were normalized to the transition $4^{+} \rightarrow 2^{+}$in Fig. 3, and to the transition $8^{+} \rightarrow 6^{+}$in Fig. 4. Note, that only some typical statistical errors are displayed. As one can see from the comparison between Fig. 3 and 4, higher beam energy leads to the large population of high-spin states. 


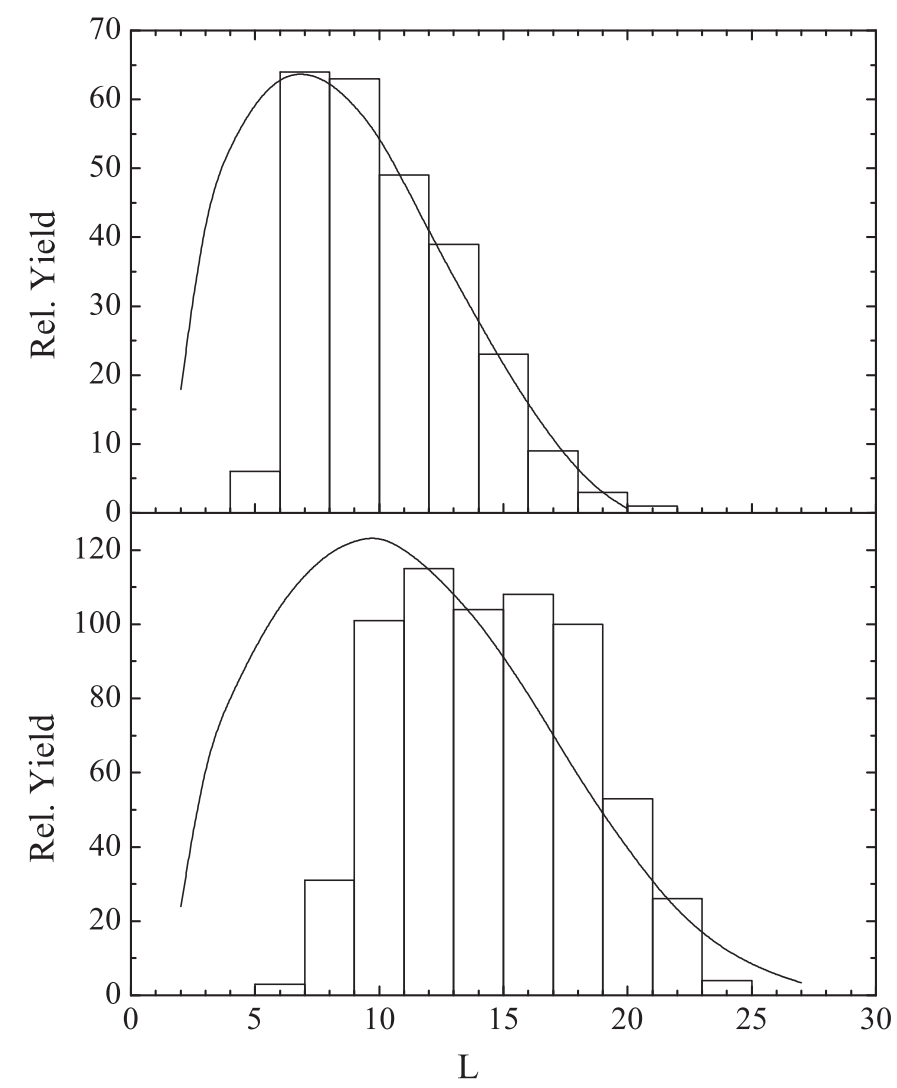

Fig. 5. The entry spin distribution of ${ }^{254} \mathrm{No}$, produced in the reaction ${ }^{208} \mathrm{~Pb}\left({ }^{48} \mathrm{Ca}, 2 n\right){ }^{254} \mathrm{No}$ at beam energies 215 $\mathrm{MeV}$ (upper part) and $219 \mathrm{MeV}$ (lower part). The experimental data from Ref. [8] are presented by squares. The calculated points are connected by lines. Damping parameter $D=1000$ is used in the calculations.)

This difference is because of the capture process: the partial capture cross section is larger at the beam energies $219 \mathrm{MeV}$ (see Fig. 1). The agreement of our results with the experiment in Figs. 3-4 seems to be quite good. The variation of the damping parameter $D$ in Eq. (19) from 600 to 1200 does not strongly affect the results of calculation. These damping parameters correspond to the widths 17-24 of the shell effects damping with the angular momentum.

The measured [8] and calculated entry spin distributions of ${ }^{254}$ No are presented in Fig. 5. The results of calculation are normalized to be equal to the experimental values in the maxima of the distributions. One can restore the entry distribution of the evaporation residue by using the population cross sections, given by Eq. (1), and taking into account the angular momentum carried out by the statistical $\gamma$-quanta. As on can see from Fig. 5, more high-spin states survive against fission with increasing $E_{c . m .}$. It could be explained by larger capture cross section at large values of the initial angular momentum (see Fig. 1). Since the results of calculations are quite insensitive to the variation of damping parameter $D$, we use $D=1000$ in Fig. 5. Our results are close to the experimental values, especially near the maxima of spin distributions.

Evaporation residue cross sections and excitation functions for (1-3)n evaporation channels have been measured in the ${ }^{48} \mathrm{Ca}+{ }^{208} \mathrm{~Pb}$ reaction in various experiments [36-39]. The experimental values and the results of our calculations using Eq. (11) and the damping parameter $D=1000$ are shown 
EPJ Web of Conferences

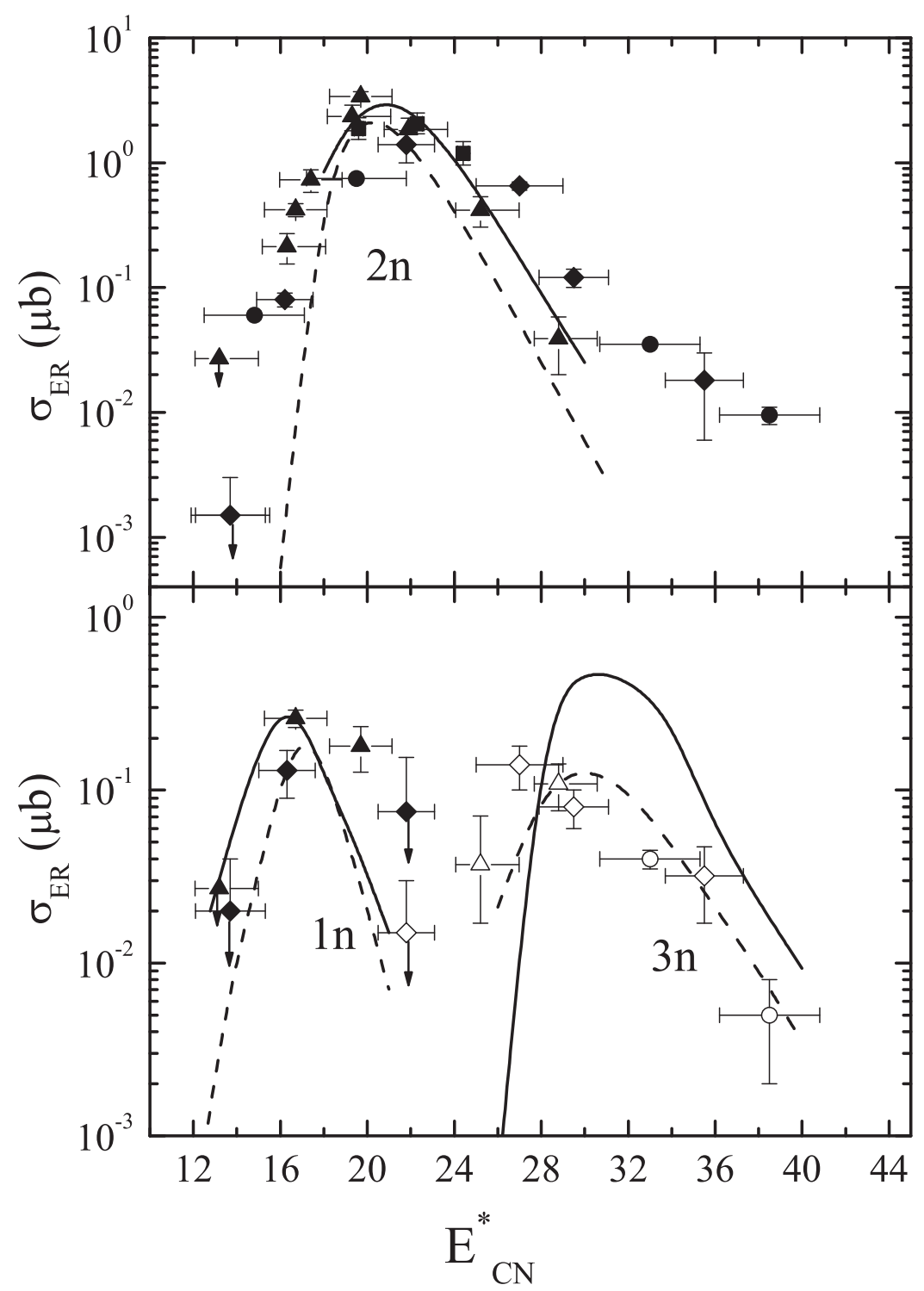

Fig. 6. Measured and calculated excitation functions for $x n$ evaporation channels in the ${ }^{48} \mathrm{Ca}+{ }^{208} \mathrm{~Pb}$ reaction. The experimental data from Refs. [36], [37], [38] and [39] are presented by circles, squares, triangles and diamonds, respectively. The solid symbols correspond to the $1 n$ and $2 n$ channels. The open symbols correspond to the $3 n$ channel. Damping parameter $D=1000$ is used in the calculations. The predictions of the properties of superheavy nuclei are taken from Ref. [18]. The results calculated in this paper and in Ref. [14] are presented by solid and dashed lines, respectively.)

in Fig. 6. The error bars represent only the statistical uncertainties. Comparing the results of various measurements shown in Fig. 6, we conclude that the systematic uncertainty in the definition of $\sigma_{E R}$ is up to a factor of 3 . The dependence of the calculated results on the parameter $D$ is rather weak. For example, in the maximum of $2 n$ evaporation channel, the variation of $D$ from 600 to 1200 leads to the variation of $\sigma_{E R}$ from 2.5 to $3.6 \mu \mathrm{b}$. The excitation functions, calculated in Ref. [14] using Eq. (12), the same parameters of Fermi-gas model and theoretical predictions of nuclear properties [18], are also presented in Fig. 6. The results, obtained with these two different approaches, are close to each other and to the experimental data. 
$\mathrm{CNR} * 11$

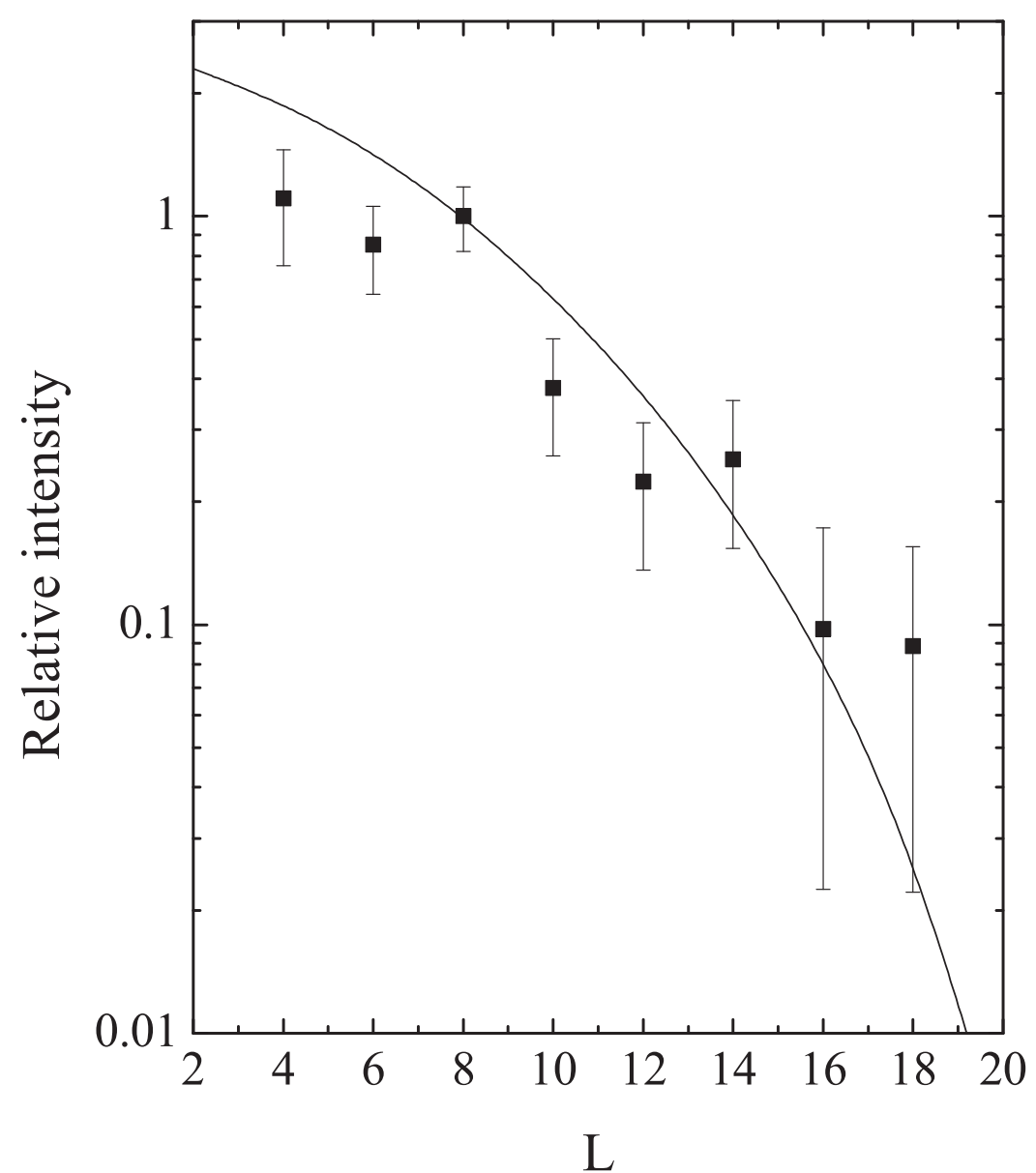

Fig. 7. Relative transition intensities in the yrast rotational band of ${ }^{252} \mathrm{No}$, produced in the reaction ${ }^{206} \mathrm{~Pb}\left({ }^{48} \mathrm{Ca}\right.$, $2 n)^{252} \mathrm{No}$ at the beam energy $215.5 \mathrm{MeV}$. Damping parameter $D=1000$ is used in the calculations. The experimental data from Refs. [9] are presented by squares. The calculated points are connected by line.)

\subsection{The residual nuclei ${ }^{252}$ No and ${ }^{250} \mathrm{Fm}$}

In Fig. 7 the measured [9] and calculated relative transition intensities in the rotational band of the nucleus ${ }^{252} \mathrm{No}$, produced in the reaction ${ }^{206} \mathrm{~Pb}\left({ }^{48} \mathrm{Ca}, 2 n\right){ }^{252} \mathrm{No}$ at the beam energy $215.5 \mathrm{MeV}$ are presented. The relative intensities were normalized to the transition $8^{+} \rightarrow 6^{+}$. The measured evaporation residue cross section $\sigma_{E R}^{\exp } \approx 220 \mathrm{nb}$ [9], its value, calculated with Eq. (11) and the damping parameter $D=1000, \sigma_{E R}=210 \mathrm{nb}$. As seen from Fig. 7, the agreement between measured and calculated relative transition intensities is good as well.

In Fig. 8 the measured [10] and calculated relative transition intensities in the rotational band of the nucleus ${ }^{250} \mathrm{Fm}$, produced in the reaction ${ }^{204} \mathrm{Hg}\left({ }^{48} \mathrm{Ca}, 2 n\right)^{250} \mathrm{Fm}$ at the beam energy $209 \mathrm{MeV}$ are presented. The relative intensities were normalized to the transition $12^{+} \rightarrow 10^{+}$. The measured evaporation residue cross section $\sigma_{E R}^{\exp }=980_{-160}^{+160} \mathrm{nb}$ [10], its value, calculated with Eq. (11) and the damping parameter $D=1000, \sigma_{E R}^{\exp }=790 \mathrm{nb}$. As seen from Fig. 8, the agreement between measured and calculated relative transition intensities is quite good in high-spin region, at $L \geq 12$. The saturation of transitions at smaller spins, observed in the experiment, can not be explained within our model. Although the disagreement at low spins is within a factor of 2. 
EPJ Web of Conferences

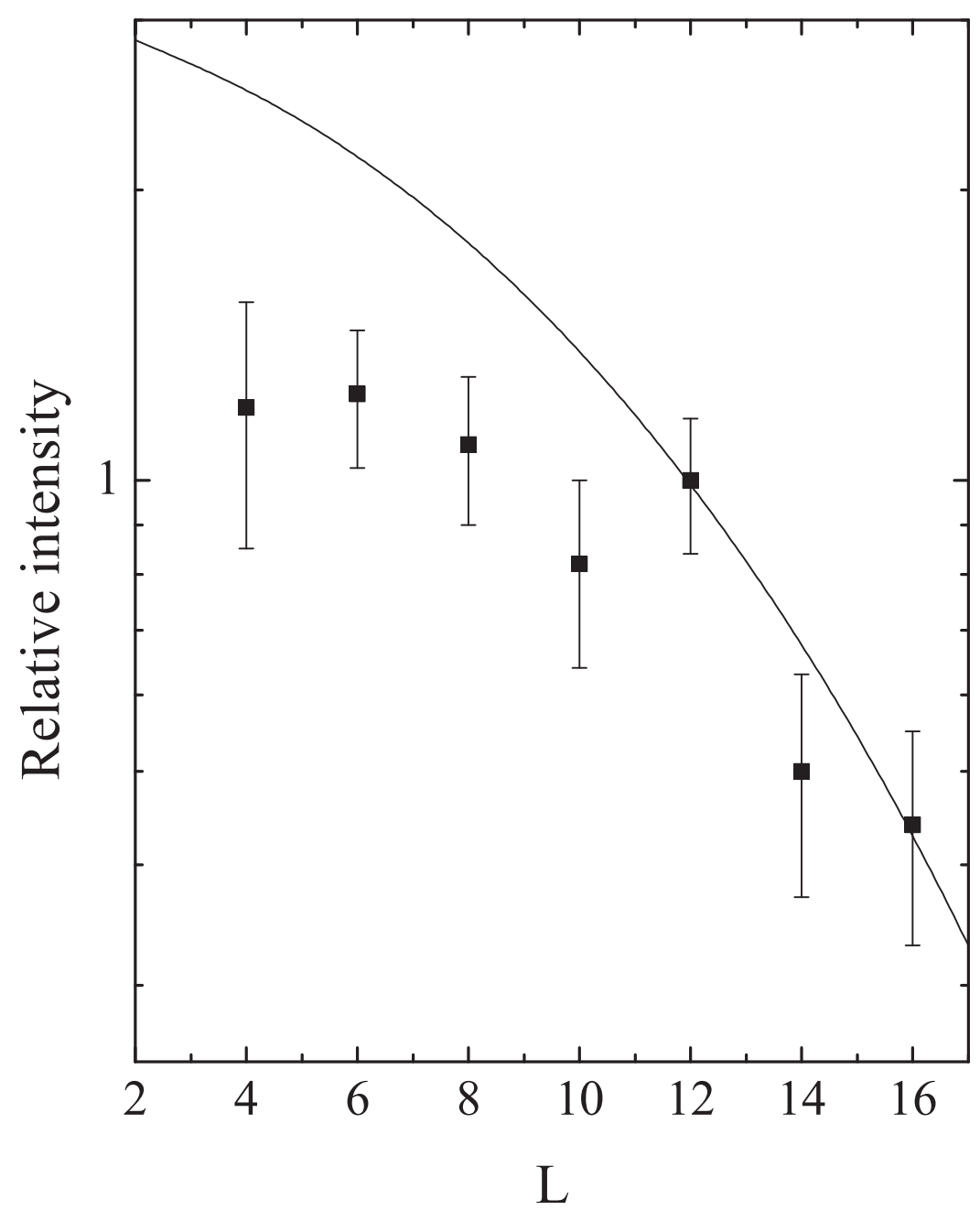

Fig. 8. Relative transition intensities in the yrast rotational band of ${ }^{250} \mathrm{Fm}$, produced in the reaction ${ }^{204} \mathrm{Hg}\left({ }^{48} \mathrm{Ca}\right.$, $2 n)^{250} \mathrm{Fm}$ at the beam energy $209 \mathrm{MeV}$. Damping parameter $D=1000$ is used in the calculations. The experimental data from Refs. [10] are presented by squares. The calculated points are connected by line.)

\section{Summary}

Using the statistical and quantum diffusion approaches, we studied the population of rotational bands in superheavy nuclei produced in fusion-evaporation reactions ${ }^{206,208} \mathrm{~Pb}\left({ }^{48} \mathrm{Ca}, 2 n\right){ }^{252,254} \mathrm{No}$ and ${ }^{204} \mathrm{Hg}\left({ }^{48} \mathrm{Ca}\right.$, $2 n)^{250} \mathrm{Fm}$. The Fermi-gas model was applied for calculating the level densities. For the description of the capture process, we used the quantum diffusion approach based on the formalism of reduced density matrix. The interval $D=600-1000$ for the damping parameter was found for the description of the damping of the shell effects with the angular momentum and used in the calculations of the relative transition intensities in the ground state rotational bands and the entry spin distributions of the evaporation residues and the evaporation residue cross sections for all considered reactions. Angular momentum dependence of these observables mainly comes from the partial capture and survival probabilities. The calculated results are in a good agreement with the experimental data.

This work was supported by DFG, RFBR, and by the Grant of the President of RF. 


\section{$\mathrm{CNR} * 11$}

\section{References}

1. A. Sobiczewski, F.A. Gareev, and B.N. Kalinkin, Phys. Lett. B22 (1966) 500; W.D. Myers and W.J. Swiatecki, Nucl. Phys. 81, (1966) 1; U. Mosel and W. Greiner, Z. Phys. A222 (1969) 261; F.O. Fiset and J.R. Nix, Nucl. Phys. A193, (1972) 647; S.G. Nilsson et al., Nucl. Phys. A115 (1968) 545; J. Randrup et al., Phys. Rev. C13, (1976) 229; P. Möller and R.J. Nix, J. Phys. G: Nucl. Part. Phys. 20, (1994) 1681; A. Sobiczewski, Physics of Particles and Nuclei, 25, (1994) 295.

2. J. Decharge, J.F. Berger, and K. Dietrich, M.S. Weiss, Phys. Lett. B451, (1999) 275.

3. M. Bender et al., Phys. Lett. B515, (2001) 42.

4. P.G. Reinhard, Rep. Prog. Phys. 52, (1989) 439; P. Ring, Prog. Part. Nucl. Phys. 37, (1996) 193.

5. S. Cwiok, et al., Nucl. Phys. A611, (1996) 211.

6. M. Bender et al., Phys. Rev. C60, (1999) 034304.

7. P. Reiter et al., Phys. Rev. Lett. 82, (1999) 509.

8. P. Reiter et al., Phys. Rev. Lett. 84, (2000) 3542.

9. R-D. Herzberg et al., Phys. Rev. C65, (2001) 014303.

10. J.E. Bastin et al., Phys. Rev. C73, (2006) 024308; P.T. Greenlees et al., Phys. Rev. C78, (2008) 021303.

11. G.G. Adamian, N.V. Antonenko, and W. Scheid, Nucl. Phys. A 678, (2000) 24.

12. G.G. Adamian, N.V. Antonenko, S.P. Ivanova, and W. Scheid, Nucl. Phys. A 646, (1999) 29.

13. A.S. Zubov, G.G. Adamian, N.V. Antonenko, S.P. Ivanova, and W. Scheid, Phys. Rev. C65, (2002) 024308.

14. A.S. Zubov, G.G. Adamian, N.V. Antonenko, S.P. Ivanova, and W. Scheid, Eur. Phys. J. A23, (2005) 249.

15. P. Möller and R. Nix, At. Data Nucl. Data Tables 39, (1988) 213.

16. P. Möller et al., At. Data Nucl. Data Tables 59, (1995) 185.

17. R. Smolanczuk, J. Skalski, and A. Sobiczewski, Phys. Rev. C52, (1995) 1871; R. Smolanczuk, Phys. Rev. C59, (1999) 2634.

18. O. Parkhomenko, I. Muntian, Z. Patyk, and A. Sobiczewski, Acta Phys. Pol. B34, (2003) 2153; I. Muntian, S. Hofmann, Z. Patyk, and A. Sobiczewski, Acta Phys. Pol. B34, (2003) 2073; I. Muntian, Z. Patyk, and A. Sobiczewski, Acta Phys. Pol. B34, (2003) 2141; I. Muntian, Z. Patyk, and A. Sobiczewski, Acta Phys. Pol. B32, (2001) 691.

19. K.-H. Schmidt and W. Morawek, Rep. Prog. Phys. 54, (1991) 949.

20. A.V. Ignatyuk, Statistical Properties of Excited Atomic Nuclei (Energoatomizdat, Moscow, 1983).

21. A.S. Zubov, V.V. Sargsyan, G.G. Adamian, and N.V. Antonenko, Phys. Rev. C, to be published.

22. N.V. Antonenko, E.A. Cherepanov, A.K. Nasirov, V.P. Permjakov, and V.V. Volkov, Phys. Lett. B 319, (1993) 425; Phys. Rev. C 51, (1995) 2635.

23. G.G. Adamian, N.V. Antonenko, W. Scheid, and V.V. Volkov, Nucl. Phys. A 633, (1998) 409; Nuovo Cimento A 110, (1997) 1143.

24. E.A. Cherepanov, A.S. Iljinov, and M.V. Mebel, J. Phys. G: Nucl. Phys. 9, (1983) 931.

25. E.A. Cherepanov, in Proc. Int. Symp. on In-Beam Nuclear Spectropscopy, (Debrecen, 1984) p.499; E.A. Cherepanov and A.S. Iljinov, Nucleonika 25, (1980) 611; E.A. Cherepanov, Preprint JINR, E7-99-27 (1999).

26. R. Vandenbosch and J.R. Huizenga, Nuclear Fission (Academic Press, New York, 1973).

27. V.V. Sargsyan, G.G. Adamian, N.V. Antonenko, and W. Scheid, Eur. Phys. J. A 45, (2010) 125; V.V. Sargsyan, G.G. Adamian, N.V. Antonenko, W. Scheid, and H.Q. Zhang, Eur. Phys. J. A 47, (2011) 38; J. of Phys.: Conf. Ser. 282 (2011) 012001.

28. J. Gilat, Phys. Rev. C 1, 1432 (1970).

29. O.V. Grusha et al., Nucl. Phys. A 429, 313 (1984).

30. O.V. Grusha, S.P. Ivanova, and Yu.N. Shubin, VANT, Nuclear Constants 1, 36 (1987).

31. A.V. Ignatyuk, K.K. Istekov, and G.N. Smirenkin, Sov. J. Nucl. Phys. 29, (1979) 875.

32. G. Audi, A.H. Wapstrab, and C. Thibault, Nucl. Phys. A 729, (2003) 337.

33. S.G. Mashnik, A.J. Sierk and K.K. Gudima, arXiv:nucl-th/0208048 (2002).

34. A.J. Sierk, Phys. Rev. C 33, (1986) 2039. 
EPJ Web of Conferences

35. W.D. Myers and W.J. Swiatecki, Phys. Rev. C 60, (1999) 014606; Nucl. Phys. A 601, (1996) 141;

Preprint LBL-36803 (1994).

36. A.V. Belozerov et al., Eur. Phys. J. A 16, (2003) 447.

37. Yu.Ts. Oganessian et al., Phys. Rev. C 64, (2001) 054606.

38. H.W. Gäggeler et al., Nucl. Phys. A 502, (1989) 561.

39. A.V. Yeremin et al., JINR Rapid Commun. No. 6[92]-98, (1998) 21. 